\title{
Compact Plasmonic Distributed-Feedback Lasers as Dark Sources of Surface Plasmon Polaritons
} \author{
Ario Cocina,,$^{\dagger}$ Aurelio A. Rossinelli,,$^{\dagger}$ Freddy T. Rabouw, ${ }^{\dagger, \xi}$ and David J. Norris ${ }^{*}{ }^{\dagger}$ \\ †Optical Materials Engineering Laboratory, \\ Department of Mechanical and Process Engineering, \\ ETH Zurich, 8092 Zurich, Switzerland \\ $\S_{\text {Debye Institute for Nanomaterials Science, }}$ \\ Utrecht University, 3584 CC Utrecht, The Netherlands
}

Raphael Brechbühler,,$^{\dagger}$ Sander J. W. Vonk, ${ }^{\dagger}$, Marianne Aellen, ${ }^{\dagger}$ Nolan Lassaline, ${ }^{\dagger}$ Robert C. Keitel, ${ }^{\dagger}$

\section{S1. SAMPLE FABRICATION}

Materials. 1000- $\mu \mathrm{m}$-thick, four-inch-diameter, single-side-polished, single-crystalline Si(100) wafers were purchased from Silicon Quest. Acetone (Technic France, Micropur VLSI Grade), isopropyl alcohol (IPA, Technic France, Micropur VLSI Grade), CSAR resist (AR-P 6200.04, Allresist GmbH), developer solution (AR 600-546, Allresist GmbH), and buffered hydrofluoric acid (hydrogen fluoride/ammonium fluoride 1:7, Technic France, Micropur VLSI Grade) used for the fabrication of Si templates were provided by the Binnig and Rohrer Nanotechnology Center (BRNC) at IBM Zurich. Sulfuric acid $\left(\mathrm{H}_{2} \mathrm{SO}_{4}, 95-98 \%\right)$, hydrogen peroxide $\left(\mathrm{H}_{2} \mathrm{O}_{2}, 30 \%\right.$, AnalaR Normapur), and nitric acid $\left(65 \% \mathrm{HNO}_{3}\right)$, were purchased from Sigma-Aldrich, VWR, and Fisher Chemicals, respectively. A MilliQ Advantage A10 water purification system was used to obtain 18.2-M $\Omega$ deionized (DI) water. Silver (Ag, 99.999\%) pellets and tungsten boats were bought from Kurt Lesker and Umicore, respectively. 1-mm-thick glass slides were purchased from Paul Marienfeld and ultraviolet-curable epoxy (OG142-95) was obtained from Epoxy Technology. Poly(methyl methacrylate) (PMMA, average molecular weight $996 \mathrm{~kg} / \mathrm{mol}$ ) and anisole (ReagentPlus, 99\%) were purchased from SigmaAldrich. 
Fabrication of the Si Templates. A four-inch Si wafer was diced into $2 \times 2-\mathrm{cm}^{2}$ chips. Each chip was cleaned by ultrasonication for $2 \mathrm{~min}$ in acetone and in IPA, and blown dry with $\mathrm{N}_{2}$. Then, the chip was pre-baked for $10 \mathrm{~min}$ at $180{ }^{\circ} \mathrm{C}$ on a hotplate and subsequently cooled for 1 min on a metal plate. A layer of AR-P 6200.04 resist was spin-coated onto the chip by accelerating at $500 \mathrm{rpm} \mathrm{s}^{-1}$ to $500 \mathrm{rpm}$ for $5 \mathrm{~s}$ and then at $2000 \mathrm{rpm} \mathrm{s}^{-1}$ to $2000 \mathrm{rpm}$ for $55 \mathrm{~s}$. The chip was post-baked for $5 \mathrm{~min}$ at $150{ }^{\circ} \mathrm{C}$. Grating structures with varying parameters were defined using electron-beam lithography (Vistec, NFL 5). After exposure, the resist was developed in the developer solution for $1 \mathrm{~min}$, and the sample was rinsed in IPA and blown dry with $\mathrm{N}_{2}$. The pattern in the resist was then transferred into the $\mathrm{Si}$ substrates with reactive-ion etching (Oxford Instruments, Plasmalab System 100) using $40 \mathrm{sccm} \mathrm{HBr}$ at a chamber pressure of 3 mTorr, with a forward power of $200 \mathrm{~W}$, a radio-frequency power of $20 \mathrm{~W}$, and an etch rate of $\sim 30 \mathrm{~nm} \min ^{-1}$. The etching time was varied on subsequent chips to achieve different etching depths. Then, the resist was removed by ultrasonication in acetone and in IPA, followed by 2 min of $\mathrm{O}_{2}$-plasma cleaning at $600 \mathrm{~W}$, a dip in buffered hydrofluoric acid, and rinsing with DI water.

Evaporation of Ag. Before the evaporation, the templates were cleaned for $\sim 20 \mathrm{~min}$ in piranha (1:1 mixture of $\mathrm{H}_{2} \mathrm{SO}_{4}: \mathrm{H}_{2} \mathrm{O}_{2}$; caution: this solution reacts violently with solvents and other organic material), ultrasonicated in DI water and in IPA, and blown dry with $\mathrm{N}_{2}$. The templates were loaded into a thermal evaporator (Kurt J. Lesker, Nano 36) onto a custom-built rotational mount tilted by $\sim 30^{\circ}$ with respect to the evaporation direction. The evaporator chamber was evacuated to a pressure below $4 \times 10^{-7} \mathrm{mbar}$, and then approximately $700 \mathrm{~nm}$ of $\mathrm{Ag}$ was evaporated onto the templates at a rate of $25 \AA \mathrm{s} \mathrm{s}^{-1}$ while rotating the rotational mount at $60 \mathrm{rpm} .{ }^{\mathrm{S} 1}$ After the evaporation, a $2.2 \times 2.2-\mathrm{cm}^{2}$-sized glass slide was affixed onto the exposed Ag surface with epoxy and the epoxy was cured by exposure with an ultraviolet lamp for $2 \mathrm{~h}$. Afterwards, the Ag-epoxy-glass stack was stripped from the template, revealing a smooth Ag surface with the negative of the initial pattern in the Si template. ${ }^{\mathrm{S} 2}$ The Si template could be reused by repeating the process described in this paragraph after removing residual Ag from the template in dilute nitric acid and rinsing with DI water.

Spin-Coating of PMMA onto Ag Surfaces. PMMA was spin-coated onto the Ag samples to study the effect of a thin dielectric layer on the plasmonic dispersion (Figure 1b-d,f,g in the main text). To 
prepare a PMMA stock solution, 2 weight percent of PMMA was dissolved in anisole by stirring for $1 \mathrm{~h}$ on a hotplate at $70^{\circ} \mathrm{C}$. PMMA was spin-coated onto the grating structures by accelerating at $500 \mathrm{rpm} \mathrm{s}^{-1}$ to $3000 \mathrm{rpm}$ for $60 \mathrm{~s}$. The sample was baked for $1 \mathrm{~min}$ on a hotplate at $110^{\circ} \mathrm{C}$.

Adding NPL Films to the Ag Surfaces. Colloidal nanoplatelets (NPLs) with graded shells of $\mathrm{Cd}_{x} \mathrm{Zn}_{1-x} \mathrm{~S}$ were drop-casted onto the $\mathrm{Ag}$ samples without PMMA as the gain material for the lasing experiments. A detailed description of the synthesis procedure and a characterization of the NPLs is given in Ref. S3 (labeled there as "4-ML-CdSe/Cdx $\mathrm{Zn}_{1-\mathrm{x}} \mathrm{S}$ "). The stock dispersion was stored in hexane at an optical density of 9 at the lowest-energy excitonic peak measured for an optical path length of $1 \mathrm{~cm}$. The quantum yield and the colloidal stability of the used batch did not deteriorate while storing the stock dispersion for more than a year. Before drop-casting, 5 parts of the stock dispersion were diluted with 5 parts of hexane and 1 part octane by volume. $40 \mu \mathrm{L}$ of the diluted NPL dispersion were drop-casted onto the $\sim 2.2 \times 2.2 \mathrm{~cm}^{2}$-sized Ag-epoxy-glass sample and slowly dried under ambient conditions.

\section{S2. MODELING}

Calculation of the Grating Dispersion. We calculated the dispersion of Ag gratings covered with a thin layer of PMMA under the assumption that the grating ridges are a weak perturbation for SPPs on a flat interface. Below, we restrict our analysis to SPPs propagating along the $\pm x$ direction (perpendicular to the grating ridges), as this is experimentally probed by our reflectivity measurements. First, we calculated the SPP dispersion for a flat Ag-dielectric-air interface. Then, the interaction with the grating is introduced by considering SPP-photon coupling according to eq 1 of the main text while retaining the SPP dispersion from a flat interface. Finally, SPP-SPP coupling around the $g_{2}$ stop gap is considered with a coupled-mode model.

SPP Dispersion: A flat Ag-dielectric-air stack supports SPPs with transverse-magnetic (TM) polarization. The real part of the in-plane wavevector, $\boldsymbol{k}_{\mathrm{SPP}}$, has a magnitude of $k_{\mathrm{SPP}}=k_{0} n_{\mathrm{SPP}}(\omega)$, where $k_{0}=\omega / c$ is the free-space wavevector of photons, $\omega$ is the angular frequency, $c$ the speed of light, and $n_{\mathrm{SPP}}(\omega)$ the real part of the frequency-dependent effective mode index of the plasmonic mode. We calculated $n_{\mathrm{SPP}}(\omega)$ for a flat three-layer geometry by solving Maxwell's equations with 
boundary conditions at the interfaces following Ref. S4. The frequency-dependent refractive index of

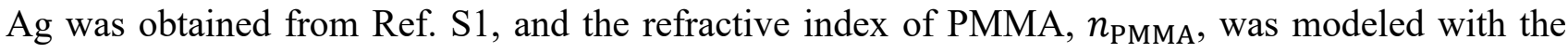
Sellmeier equation

$$
n_{\mathrm{PMMA}}^{2}(\lambda)=1+\frac{B_{1} \lambda^{2}}{\lambda^{2}-C_{1}}
$$

with the Sellmeier coefficients $B_{1}=1.1819$ and $C_{1}=0.011313 \mu \mathrm{m}^{2}$ from Ref. S5 (fit of the experimental data with the Sellmeier dispersion formula by Mikhail Polyanskiy) and the wavelength of light $\lambda$.

Uncoupled Grating Dispersion: The calculated SPP dispersion (red lines in Figure 1b in the main text) was inserted into eq 1 of the main text to obtain the uncoupled grating dispersion (black dashed lines in Figure $1 \mathrm{~b}$ in the main text). We found the best match to our experimental data when we set the PMMA-layer thickness to $50 \mathrm{~nm}(52.5 \mathrm{~nm})$ for gratings with ridge heights of 8 and $13 \mathrm{~nm}(20 \mathrm{~nm})$.

Coupled-Mode Model: Stop gaps in the SPP dispersion occur when the grating diffraction couples counter-propagating SPP modes (see eq 2 in the main text). The coupling introduces a hybridization of the propagating modes, and the coupled modes feature standing-wave character at the stop-gap edges. The solutions at the upper- and lower-energy edges feature a different spatial localization within the unit cell of the periodic grating with different associated energies. ${ }^{\mathrm{S} 6}$

Here, we focus on the stop gap occurring due to SPP-SPP coupling by $g_{2}$. Because we cannot probe the SPP dispersion directly with our reflectivity measurements, we apply the coupled-mode formalism to the branches that are shifted into the light cone after diffraction by $\pm g_{1}$. The in-plane wavevectors $k_{x}$ of the uncoupled modes are given by

$$
k_{x,+}(\omega)=k_{\mathrm{SPP}}(\omega)-g_{1}
$$

and

$$
k_{x,-}(\omega)=-k_{\mathrm{SPP}}(\omega)+g_{1}
$$

We numerically solved eqs S2 and S3 for the uncoupled mode energies $\hbar \omega_{+}\left(k_{x}\right)$ and $\hbar \omega_{-}\left(k_{x}\right)$. The energies of the coupled modes are given by the eigenvalues of the interaction matrix

$$
\mathbf{H}\left(k_{x}\right)=\hbar\left(\begin{array}{cc}
\omega_{+}\left(k_{x}\right) & \Gamma_{g_{2}} \\
\Gamma_{g_{2}} & \omega_{-}\left(k_{x}\right)
\end{array}\right)
$$


where $\Gamma_{g_{2}}$ is the SPP-SPP coupling rate at the $g_{2}$ stop gap. ${ }^{\mathrm{S} 7}$ We fitted $\Gamma_{g_{2}}$ to find the best overlap between the dispersion of the coupled modes (red dashed lines in Figure 1 $\mathrm{c}$ in the main text) and the experimental reflectivity dips (blue areas in Figure $1 \mathrm{c}$ in the main text). For a given $\Gamma_{g_{2}}$ the experimental reflectivity values evaluated at the positions of the coupled modes (i.e. at their energies and corresponding $k_{x}$ values) were summed up. Then, the optimum $\Gamma_{g_{2}}$ was found that minimized this reflectivity sum. The fitted values of $\Gamma_{g_{2}}$ are plotted in Figure 1d in the main text.

Lasing-Mode Profiles Calculated with Coupled-Wave Theory. We used scalar coupled-wave theory for DFB lasers, as introduced by Kogelnik and Shank, ${ }^{\mathrm{S} 8}$ to model the lasing-mode profiles on the plasmonic $g_{2}$-feedback cavities. The leakage from the lasing modes into the far field by Bragg diffraction is then calculated in real- and $k$-space.

Coupled-wave theory considers counter-propagating plane waves with electric fields given by $R(x) \exp \left(-\mathrm{i} k_{\mathrm{SPP}} x\right)$ and $S(x) \exp \left(\mathrm{i} k_{\mathrm{SPP}} x\right)$, travelling in the $+x$ and the $-x$ directions, respectively, with smoothly varying envelopes $R(x)$ and $S(x)$. The plane waves are subject to gain and weak periodic scattering at the grating ridges, leading to coupling between the waves close to the stop-gap edge. Coupled-wave theory provides solutions for $R(x)$ and $S(x)$ of the form

$$
R(x)=\sinh \left[\gamma\left(x+\frac{L}{2}\right)\right]
$$

and

$$
S(x)= \pm \sinh \left[\gamma\left(x-\frac{L}{2}\right)\right]
$$

such that the total electric field in the feedback cavity

$$
E(x)=R(x) \exp \left(-\mathrm{i} k_{\mathrm{SPP}} x\right)+S(x) \exp \left(\mathrm{i} k_{\mathrm{SPP}} x\right)
$$

fulfills the governing Helmholtz equation (see Ref. S8). $\gamma=\sqrt{\kappa^{2}+(\alpha+i \delta)^{2}}$ is defined through a parameter set consisting of a gain threshold $\alpha$, detuning $\delta$ (describing the departure of the lasing-mode frequency from the center of the stop gap), and coupling strength $\kappa$, which simultaneously need to fulfill the self-consistency equations

$$
\begin{gathered}
\kappa \mp \operatorname{Im}\left(\frac{\gamma}{\sinh \gamma L}\right)=0 \\
\operatorname{Re}\left(\frac{\gamma}{\sinh \gamma L}\right)=0
\end{gathered}
$$




$$
\begin{aligned}
& \alpha-\operatorname{Re}(\gamma \operatorname{coth} \gamma L)=0 \\
& \delta+\operatorname{Im}(\gamma \operatorname{coth} \gamma L)=0
\end{aligned}
$$

Figure S6a plots solutions to the individual eqs S8 to S11 for a cavity length $L=9.46 \mu \mathrm{m}$, as in the experiment, and a coupling strength $\kappa=0.25 \mu \mathrm{m}^{-1}$. This coupling strength is obtained from our experimentally measured $g_{2}$-feedback rate, $\Gamma_{g_{2}}$, as $\kappa=\Gamma_{g_{2}} n_{\mathrm{SPP}} / c$ with the experimentally determined effective mode index of SPPs, $n_{\mathrm{SPP}}=1.48$ (see main text). The intersections of the individual lines in Figure S6a (highlighted with black circles) give the self-consistent sets of parameters that fulfill the Helmholtz equation. Multiple solutions are found on both sides of the stop-gap center (i.e. at positive and negative detunings). Here, we consider only the modes with the lowest gain threshold of $\alpha=0.087 \mu \mathrm{m}^{-1}$ at detunings $\delta= \pm 0.386 \mu \mathrm{m}^{-1}$. The solution with positive (negative) detuning corresponds to lasing at the higher-energy (lower-energy) stop-gap edge. The lasing-mode profiles observed in the far field are determined by the slowly varying envelopes $R(x)$ and $S(x)$ and given by

$$
I(x)=|R(x)+S(x)|^{2}
$$

The solutions for $S(x)$ at the two stop-gap edges have opposite signs (see eq S6 and Figure S6a), resulting in qualitatively different lasing-mode profiles observed in the far field; at the dark (bright) stop-gap edge $R(x)$ and $S(x)$ partially cancel (add up). ${ }^{\mathrm{S} 9}$ The resulting differences will be discussed in more details further below.

In the lasing experiments presented in Figures 2 and 3 in the main text, we only observe the lasing mode at the dark stop-gap edge. The partial cancellation of the diffracted signal in the far field reduces photon leakage for the lasing mode at the dark edge compared to the bright one, resulting in a lower lasing threshold (not considered in the coupled-wave treatment here). Combined with the better overlap of the dark stop-gap edge with the gain envelope of the gain material, this could explain the absence of plasmonic lasing at the bright stop-gap edge.

In Figure 3e in the main text we plot the coupled-wave solution (eq S12) at the dark stop-gap edge in real space. From the convolution theorem, it follows that the slowly varying function $R(x)+S(x)$ defines the line shape in Fourier space. ${ }^{\text {S10 }}$ Hence, to obtain the intensity distribution of the lasing mode 
diffracted into the light cone by $g_{1}$ in $k$-space (Figure $3 \mathrm{~h}$ in the main text), we propagate $R(x)+S(x)$ to $k$-space by a Fourier transform, $\mathcal{F}$, as $I\left(k_{x}\right)=I_{0}|\mathcal{F}[R(x)+S(x)]|^{2}$.

Now, we study the influence of the coupling strength on the gain thresholds and the lasing-mode profiles. To do so, we find self-consistent coupled-wave solutions for different values of $\kappa=\{0.0025,0.005,0.025,0.05,0.25,0.5\} \mu \mathrm{m}^{-1}$ and again only consider the solutions with the lowest gain threshold. We observe that the gain threshold decreases and the detuning increases for increasing coupling strength (Figure S6b). Hence, feedback cavities with larger coupling rates (corresponding to larger stop-gap widths) feature a lower lasing threshold. Figure S6c shows the realspace lasing-mode profiles observed in the far field (eq S12) as a function of increasing coupling strength from bottom to top. The product of the feedback strength and the cavity length (here, $L=9.46 \mu \mathrm{m})$ is typically used to separate the observed profiles into the undercoupled regime $(\kappa L \ll 1)$ and overcouped regime $(\kappa L \gg 1)$ with significantly different lasing-mode profiles. Undercoupled profiles feature predominantly emission at the cavity edges for both the dark (dashed lines) and bright (solid lines) stop-gap edges. In the overcoupled regime, the solution at the bright edge features the strongest intensity in the center. In Figure S6d we plot the corresponding $k$-space profiles. The dark (bright) edge features an intensity node (peak) at $k_{x}=0 \mu \mathrm{m}^{-1}$.

The good match between the $k$-space patterns predicted by coupled-wave theory and the experimental data (Figure $3 \mathrm{~h}$ in the main text) shows that the plasmonic lasing observed on the feedback cavity indeed originates from distributed feedback action and diffraction by $g_{1}$ at the dark stop-gap edge. However, Figure S6c,d highlights that the lasing-mode profile is not very sensitive to a change of the coupling strength as long as the coupling strength remains somewhat close to our experimentally determined coupling strength of $\kappa=0.25 \mu \mathrm{m}^{-1}$ (corresponding to $\kappa L \approx 2.4$ ). A more robust comparison between the below-threshold coupling strength (obtained from the stop-gap width) and the above-threshold lasing-mode profiles would ideally be based on experimental measurements with cavities of different lengths that probe both the undercoupled and overcoupled regime, as done in Ref. S9. 


\section{S3. OPTICAL MEASUREMENTS}

Reflectivity Measurements. A sketch of the optical setup used to obtain momentum-resolved reflectivity maps is depicted in Figure S4a. A sample containing plasmonic gratings covered with a thin layer of PMMA was mounted on an inverted microscope (Nikon, Eclipse Ti-U) in the focus of a microscope objective [Nikon, TU Plan Fluor, numerical aperture (NA) of 0.8]. The sample was illuminated through an excitation arm (Nikon, TI-FL) with a broadband halogen lamp (Nikon, LHSH100C-1) from all angles by imaging the filament of the lamp onto the back focal plane of the objective after reflection off a 50/50 beam splitter. The field diaphragm [an iris in the image plane of the excitation path $\left(\mathrm{I}_{\mathrm{e}}\right)$ ] was partially closed to illuminate only the area of the investigated grating on the sample. The light reflected off the sample was collected through the same microscope objective and transmitted through the beam splitter. $I_{c}$ and $F_{c}$ denote an image plane and a Fourier plane in the collection path after the lenses $\mathrm{L}_{1}$ (tube lens) and $\mathrm{L}_{2}$, respectively. The Fourier plane was relayed onto a 50- $\mu \mathrm{m}$-wide entrance slit of an imaging spectrograph (Andor, Shamrock 303i) by using $\mathrm{L}_{3}$ ("Fourier lens") and $\mathrm{L}_{4} .{ }^{\mathrm{S} 11} \mathrm{~L}_{2}, \mathrm{~L}_{3}$ (Thorlabs, AC-256-200-A, $f_{2}=f_{3}=200 \mathrm{~mm}$ ), and $\mathrm{L}_{4}$ (Thorlabs, AC-508-200$\mathrm{A}, f_{4}=200 \mathrm{~mm}$ ) are achromatic doublets. A linear polarizer (Thorlabs, LPVISE100-A) was placed in the image plane of the collection path $\left(I_{c}\right)$ to selectively transmit light with electric fields aligned with the entrance slit (p-polarized light that could couple to SPPs). The signal transmitted through the slit was a slice through $k$-space near $k_{y} / k_{0}=0$. We spectrally dispersed this signal $(150 \mathrm{lines} / \mathrm{mm}$ grating blazed at $500 \mathrm{~nm}$ ) and recorded the resulting image with a camera (Andor, Zyla 4.2 PLUS sCMOS) by accumulating 5 frames, each with $1 \mathrm{~s}$ integration time. The momentum-resolved spectra were normalized by a similarly obtained momentum-resolved reference spectrum reflected from a flat Ag/PMMA surface. An overview of the experimental data obtained is depicted in Figure S1.

SPP-Transmission Measurements. To probe the $g_{1}$ stop-gap width of plasmonic DFB cavities with SPPs, the optical setup used for reflectivity measurements (Figure S4a) was modified. A sample containing an incoupling grating, a feedback cavity, and an outcoupling grating (see sketch in Figure 1e in the main text; details on the design can be found in the Methods section of the main text) was mounted on the inverted microscope in the focus of the microscope objective. SPPs were launched at 
the incoupling grating by illumination with the broadband halogen lamp. The field diaphragm at $\mathrm{I}_{\mathrm{e}}$ was closed to selectively illuminate the incoupling grating. The angular content of the illumination light was adjusted to only launch SPPs propagating along the $x$ direction by imaging a custom rectangular mask placed in the Fourier plane of the excitation path $\left(\mathrm{F}_{\mathrm{e}}\right)$ onto the back focal plane of the objective, transmitting only light with $k_{y} \approx 0 \mu \mathrm{m}^{-1}$ at all accessible $k_{x}$. The launched SPPs propagated over the feedback cavity, and the transmitted signal was recollected from the outcoupling grating. The sample was imaged onto the entrance slit of the imaging spectrograph [Fourier lens $\left(\mathrm{L}_{3}\right)$ removed], and an iris was inserted in the image plane of the collection path $\left(I_{c}\right)$ to block specular reflection from the incoupling grating. To obtain a transmission spectrum, we aligned the $x$ axis of the sample (see Figure 1e in the main text) along the entrance slit, spectrally dispersed the signal transmitted through the slit (150 lines/mm grating blazed at $500 \mathrm{~nm}$ ), and recorded the resulting $x$ resolved transmission spectra with the CMOS camera by accumulating 12 frames, each with $5 \mathrm{~s}$ integration time.

Despite our "dark-field" configuration (illumination only on the incoupling grating and collection only from the feedback cavity and the outcoupling grating), we observed a dim background signal on the entire collection area due to stray scattered light caused by dust/imperfections on the microscope objective. We subtracted this background signal, acquired with the same imaging conditions on a flat Ag/PMMA film, from the $x$-resolved transmission spectra (Figure S5a). Figure $1 \mathrm{f}$ in the main text and Figure S5b,e show background-corrected spectra integrated along $x$ over the length of the outscattering grating. The spectra are convoluted with the emission spectrum of the halogen lamp, the coupling efficiencies of the in- and outcoupling gratings, and the spectral dependence of the SPP propagation length. Nevertheless, by comparing the spectra obtained for feedback cavities with different duty cycles, a clear change in the stop-gap width can be observed. We defined the stop-gap edges as the energies for which the transmission signal dropped to half of the difference between the maximum and minimum transmission value of the corresponding side of the stop-gap center. From the stop-gap width (Figure $\mathrm{S} 5 \mathrm{~d}), \Delta E_{g_{1}}(d, h)$, we determined the feedback rate at the $g_{1}$ stop gap from $\Gamma_{g_{1}}=\Delta E_{g_{1}} /(2 \hbar)$ (Figure $1 \mathrm{~g}$ in the main text). The photon-SPP coupling efficiency of our shallowest 
gratings (ridge height of $8 \mathrm{~nm}$ ) was too low to acquire transmittance spectra with a reasonable signalto-noise ratio. For ridge heights of $13 \mathrm{~nm}$, we inserted a linear polarizer (Thorlabs, LPVISE100-A) directly after the image plane of the collection path $\left(\mathrm{I}_{\mathrm{c}}\right)$, selectively transmitting light with electric fields aligned with the entrance slit, to improve the signal-to-noise ratio.

Lasing Experiments. A sketch of the optical setup used for lasing measurements in real- and $k$-space is depicted in Figure S4b. A plasmonic sample covered with a thin layer of NPLs was mounted on a three-axis piezo positioning system (Attocube, $1 \times$ ANPz101 and $2 \times$ ANPx101) inside a closed-cycle helium cryostat (Montana Instruments, Cryostation 2 with low-working-distance option), cooled to $\sim 4 \mathrm{~K}$, and brought into the focus of an objective (Nikon, CFI S P-Fluor ELWD; NA of 0.7). The NPLs were optically excited at $480 \mathrm{~nm}(2.583 \mathrm{eV})$ using an excitation laser pulsed at $100 \mathrm{~Hz}$ from an optical parametric amplifier (Spectra-Physics, Spirit OPA) pumped by a pulsed laser at $1040 \mathrm{~nm}$ (SpectraPhysics, Spirit; pulse width $\sim 340 \mathrm{fs}$ ). To adjust the laser power, the collimated excitation laser beam was sent through a gradient neutral-density filter wheel (Thorlabs, NDC-50C-2M-B) on a motorized rotation stage. The beam was redirected by a 488-nm long-pass dichroic beam splitter and defocused onto the plasmonic sample using a defocusing lens ( $\mathrm{L}_{\mathrm{d}}$ with focus length $f_{\mathrm{d}}$ ) placed $f_{\mathrm{d}}$ before the back focal plane of the cryostat objective. This resulted in a Gaussian excitation profile with a 1/e radius of $\sim 32 \mu \mathrm{m}$ (see subsection "Power Calibration" below). A custom shadow mask was placed $f_{\mathrm{d}}$ before the defocusing lens to further shape the real-space excitation profile on the sample. The excitation area was restricted to excite the $10 \times 10-\mu \mathrm{m}^{2}$-sized area of the feedback cavity only, or to excite a $25 \times 20$ $\mu \mathrm{m}^{2}$-sized area that included the feedback cavity, the outscatterers, and the unstructured area between the outscatterers, as indicated in the main text. The intensity distribution over the excited areas was nearly constant for both illumination conditions (flat-top excitation) because the defocused Gaussian illumination spot was significantly larger than the illumination area. The photoluminescence from the sample was collected with the same objective and passed through the dichroic beam splitter and a 590nm long-pass filter. A $k$-space image of the emission could be obtained by using lenses $\mathrm{L}_{1}$ through $\mathrm{L}_{4}$ to image the back focal plane of the objective onto the entrance port of an imaging spectrograph (Andor, Shamrock 303i). The Fourier lens $\left(\mathrm{L}_{3}\right)$ was removed to acquire real-space images. The 
resulting images were recorded with an air-cooled electron-multiplying charge-coupled device (EMCCD) camera (Andor, iXon 888 Ultra) with an integration time of $0.5 \mathrm{~s}$ and an electronmultiplying gain of 300 .

Momentum-Resolved Emission Spectra: The Fourier lens was inserted in the collection path and the entrance slit of the imaging spectrograph was closed to $50 \mu \mathrm{m}$. The signal transmitted through the slit was a slice through $k$-space near $k_{y} / k_{0}=0$. This signal was spectrally dispersed by the spectrograph (600 lines/mm grating blazed at $500 \mathrm{~nm})$.

Images in $k$-Space: For $k$-space images of the emission at the lasing wavelength, tunable shortpass and long-pass filters (628 $\mathrm{nm}$ VersaChrome Edge, Semrock) were placed in the collection path and were tuned to act as a band-pass filter (transmission spectrally centered at the lasing peak wavelength with full-width at half-maximum of $\sim 5 \mathrm{meV}$ for Figure $3 \mathrm{a}, \mathrm{b}, \mathrm{f}$ in the main text and $\sim 6.5 \mathrm{meV}$ for Figure 4d,f in the main text and Figure S3). The Fourier lens $\left(\mathrm{L}_{3}\right)$ was inserted, and the slit and the dispersion grating were removed.

Real-Space Images: For real-space images of the emission at the lasing wavelength, the same configuration was applied as for $k$-space images, but the Fourier lens $\mathrm{L}_{3}$ was removed.

Power Calibration: For each image acquired with the EMMCD camera, we recorded a signal proportional to the excitation power on the optical table $\left(P_{\text {table }}\right)$. To do so, we redirected a fraction of the collimated excitation beam with a beam splitter after the gradient neutral-density filter wheel towards a photodiode (Thorlabs, S120VC) connected to a power meter (Thorlabs, PM100D). Additionally, we performed calibration measurements for different $P_{\text {table }}$ and recorded the corresponding power values of the defocused Gaussian excitation spot (without excitation mask inserted) at the position of the sample $\left(P_{\text {sample }}\right)$ with a second photodiode (Thorlabs S170C) and a second identical power meter. A linear fit yields the relation $P_{\text {sample }}=f\left(P_{\text {table }}\right)$. To calculate the corresponding fluences, we recorded the photoluminescence-intensity distribution of the defocused laser spot at the sample position on a flat $\mathrm{Ag} / \mathrm{NPL}$ film without the grating structures under low excitation fluence. After subtracting a constant background due to dark counts, a Gaussian intensity distribution $i(x, y)=I_{0} \exp \left[-\frac{\left(x-x_{0}\right)^{2}}{r^{2}}-\frac{\left(y-y_{0}\right)^{2}}{r^{2}}\right]$ was fitted to the measured intensity profile, where $r$ 
is the $1 / e$ radius of the Gaussian distribution, $x_{0}$ and $y_{0}$ denote the position of the beam center, and $I_{0}$ the peak intensity. The integrated intensity of the Gaussian profile equals $I_{0} A_{\mathrm{eff}}$ with $A_{\mathrm{eff}}=\pi r^{2}$, and therefore the peak fluence can be calculated as $F=P_{\text {table }} /\left(f_{\text {rep }} A_{\text {eff }}\right)$, where $f_{\text {rep }}=100 \mathrm{~Hz}$ is the repetition rate of the excitation laser. 


\section{S4. SUPPLEMENTARY REFERENCES}

(1) McPeak, K. M.; Jayanti, S. V.; Kress, S. J. P.; Meyer, S.; Iotti, S.; Rossinelli, A.; Norris, D. J. Plasmonic Films Can Easily Be Better: Rules and Recipes. ACS Photonics 2015, 2, 326-333.

(2) Nagpal, P.; Lindquist, N. C.; Oh, S.-H.; Norris, D. J. Ultrasmooth Patterned Metals for Plasmonics and Metamaterials. Science 2009, 325, 594-597.

(3) Rossinelli, A. A.; Rojo, H.; Mule, A. S.; Aellen, M.; Cocina, A.; De Leo, E.; Schäublin, R.; Norris, D. J. Compositional Grading for Efficient and Narrowband Emission in CdSe-Based Core/Shell Nanoplatelets. Chem. Mater. 2019, 31, 9567-9578.

(4) Dionne, J. A.; Verhagen, E.; Polman, A.; Atwater, H. A. Are Negative Index Materials Achievable with Surface Plasmon Waveguides? A Case Study of Three Plasmonic Geometries. Opt. Express 2008, 16, 19001.

(5) Sultanova, N.; Kasarova, S.; Nikolov, I. Dispersion Properties of Optical Polymers. Acta Phys. Pol. A 2009, 116, 585-587.

(6) Barnes, W. L.; Preist, T. W.; Kitson, S. C.; Sambles, J. R. Physical Origin of Photonic Energy Gaps in the Propagation of Surface Plasmons on Gratings. Phys. Rev. B 1996, 54, 6227-6244.

(7) van Exter, M. P.; Tenner, V. T.; van Beijnum, F.; de Dood, M. J. A.; van Veldhoven, P. J.; Geluk, E. J.; ’t Hooft, G. W. Surface Plasmon Dispersion in Metal Hole Array Lasers. Opt. Express 2013, 21, 27422.

(8) Kogelnik, H.; Shank, C. V. Coupled-Wave Theory of Distributed Feedback Lasers. J. Appl. Phys. 1972, 43, 2327-2335.

(9) Guo, K.; Koenderink, A. F. Spatial Intensity Distribution in Plasmonic Particle Array Lasers. Phys. Rev. Appl. 2019, 11, 024025.

(10) Blanchard, R.; Menzel, S.; Pflügl, C.; Diehl, L.; Wang, C.; Huang, Y.; Ryou, J.-H.; Dupuis, R. D.; Dal Negro, L.; Capasso, F. Gratings with an Aperiodic Basis: Single-Mode Emission in Multi-Wavelength Lasers. New J. Phys. 2011, 13, 113023.

(11) Kurvits, J. A.; Jiang, M.; Zia, R. Comparative Analysis of Imaging Configurations and Objectives for Fourier Microscopy. J. Opt. Soc. Am. A 2015, 32, 2082-2092. 

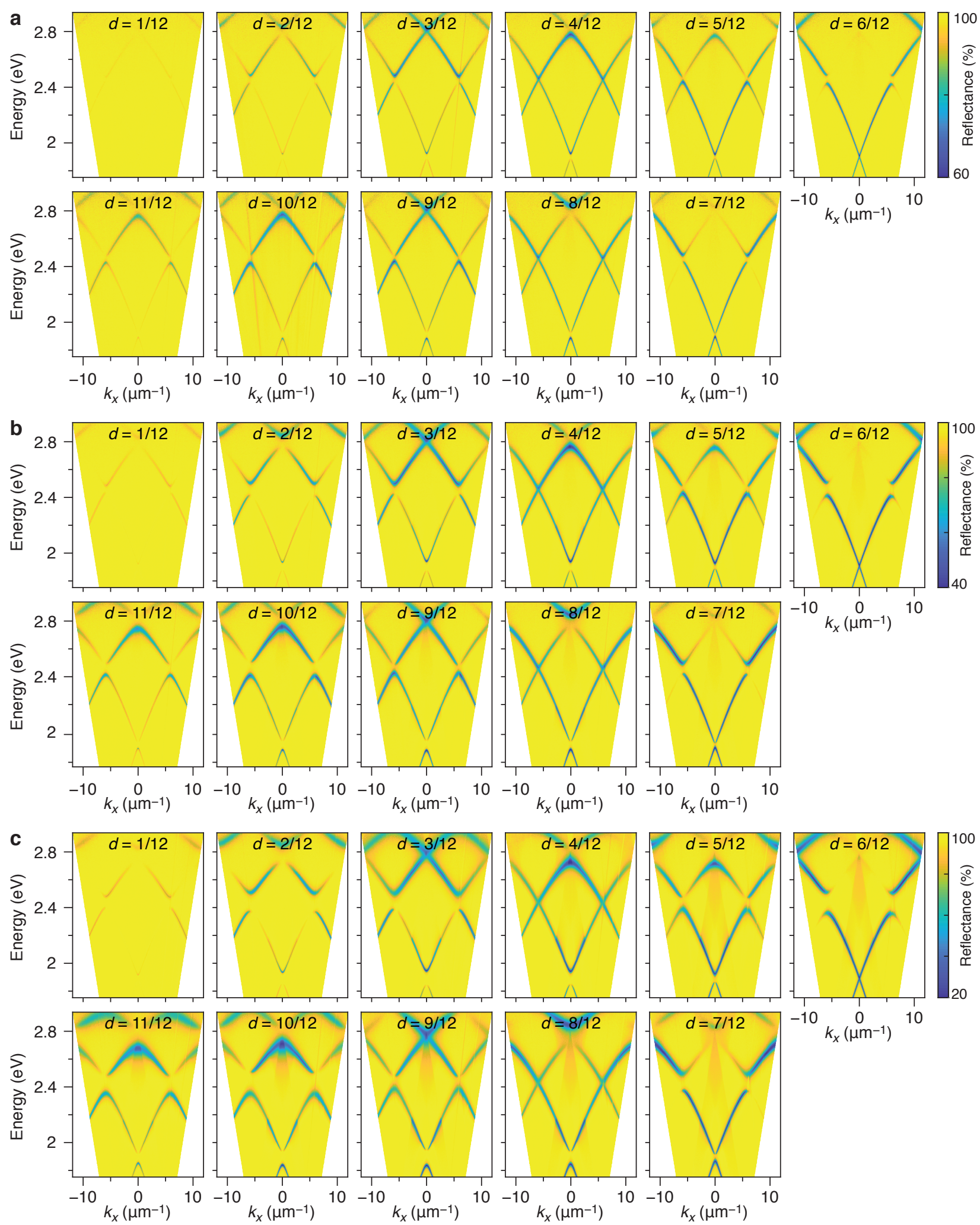

Figure S1. Momentum-resolved reflectivity spectra of $50 \times 50-\mu \mathrm{m}$-sized Ag gratings with a pitch of $560 \mathrm{~nm}$, varying duty cycles $d$, and ridge heights $h$ of (a) $8 \mathrm{~nm}$, (b) $13 \mathrm{~nm}$, and (c) $20 \mathrm{~nm}$, respectively. The gratings were covered with a $\sim 50 \mathrm{~nm}$ thick layer of PMMA. The blue streaks of lowered reflectivity correspond to photon-SPP coupling. More information about the optical setup can be found in Section S3 above. 


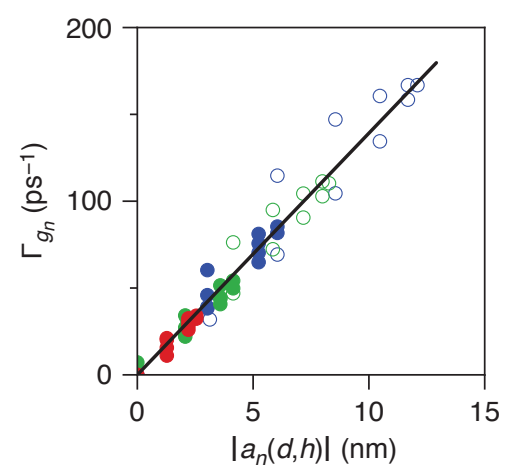

Figure S2. Feedback rates at the $g_{n}$-feedback condition, $\Gamma_{g_{n}}$, plotted against the magnitude of the Fourier coefficient, $a_{n}(d, h)$, for $n=\{1,2\}$. The filled (empty) circles correspond to the experimental data presented in Figure 1d (1g) in the main text for Ag cavities covered with 50 nm of PMMA and the $g_{2}\left(g_{1}\right)$ stop gap centered around $1.9 \mathrm{eV}$. Red, green, and blue denote cavities with ridge heights of 8,13 , and $19 \mathrm{~nm}$, respectively, and various duty cycles. The black solid line is a linear least-squares regression to the experimental data with a slope of $13.9 \mathrm{ps}^{-1} \mathrm{~nm}^{-1}$. The extracted linear relationship confirms that coupling of counter-propagating modes by $g_{n}$ depends linearly on the magnitude of the $n$-th Fourier coefficient in the grating height profile in the limit of shallow gratings $(h / \lambda \ll 1) .{ }^{\mathrm{S} 6}$ The slope is expected to increase with confinement of the SPP mode to the Ag-dielectric interface and therefore depends on the thickness and refractive index of the dielectric layer and the energetic position of the $g_{n}$ stop gap. 

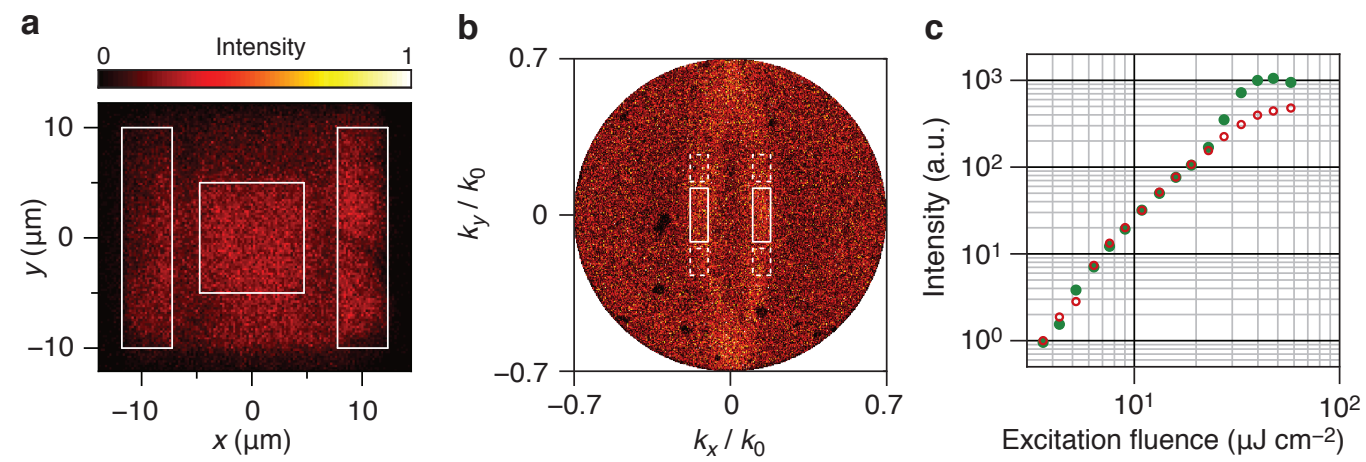

Figure S3. Below-threshold (a) real-space image (excitation fluence of $19 \mu \mathrm{J} \mathrm{cm}^{-2}$ ) and (b) $k$-space image $\left(23 \mu \mathrm{J} \mathrm{cm}^{-2}\right)$ at the lasing wavelength for the $g_{1}$-feedback cavity from Figure 4 in the main text. The images are spectrally filtered with a band pass around the lasing wavelength (see main text). The white square and rectangular boxes in (a) indicate the positions of the feedback cavity and outscattering gratings, respectively. The two faint arcs in (b) correspond to the SPP dispersion diffracted into the light cone by $\pm g_{1}$ on the outscattering gratings. (c) Intensity of the lasing signal (background signal) diffracted by the outscattering gratings plotted as green solid circles (red circles) integrated over the white solid (white dashed) boxes from (b) as a function of the excitation fluence.
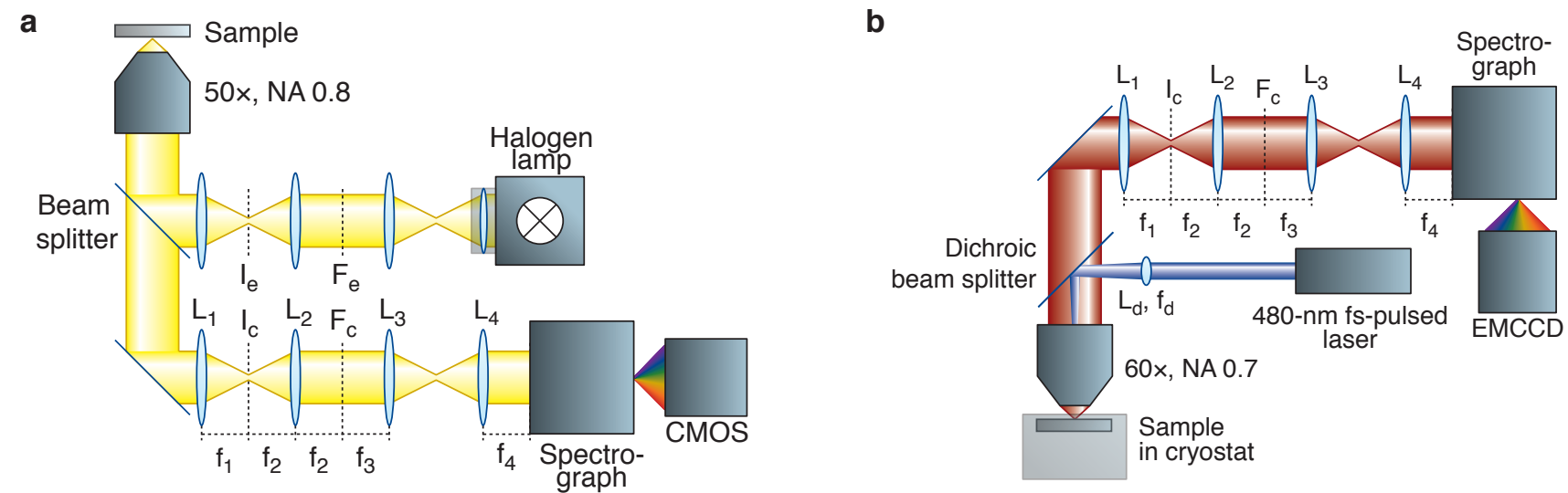

Figure S4. Schematics of the optical setups. (a) Setup used for reflectivity measurements and SPPtransmission measurements. (b) Setup used for lasing experiments. Both sketches show the configuration for imaging in $k$-space. Lenses $\mathrm{L}_{3}$ and $\mathrm{L}_{4}$ form a lens pair to image the Fourier plane of the collection path $\left(\mathrm{F}_{\mathrm{c}}\right.$; an image of the back focal plane of the microscope objective) onto the entrance slit of the imaging spectrograph. ${ }^{\mathrm{S} 11}$ A real-space image of the sample is retrieved by removing the Fourier lens $\mathrm{L}_{3}$. $\mathrm{L}_{i}$ and $f_{i}$ denote lenses and their corresponding focal lengths, respectively. See Section S3 above for further details. 

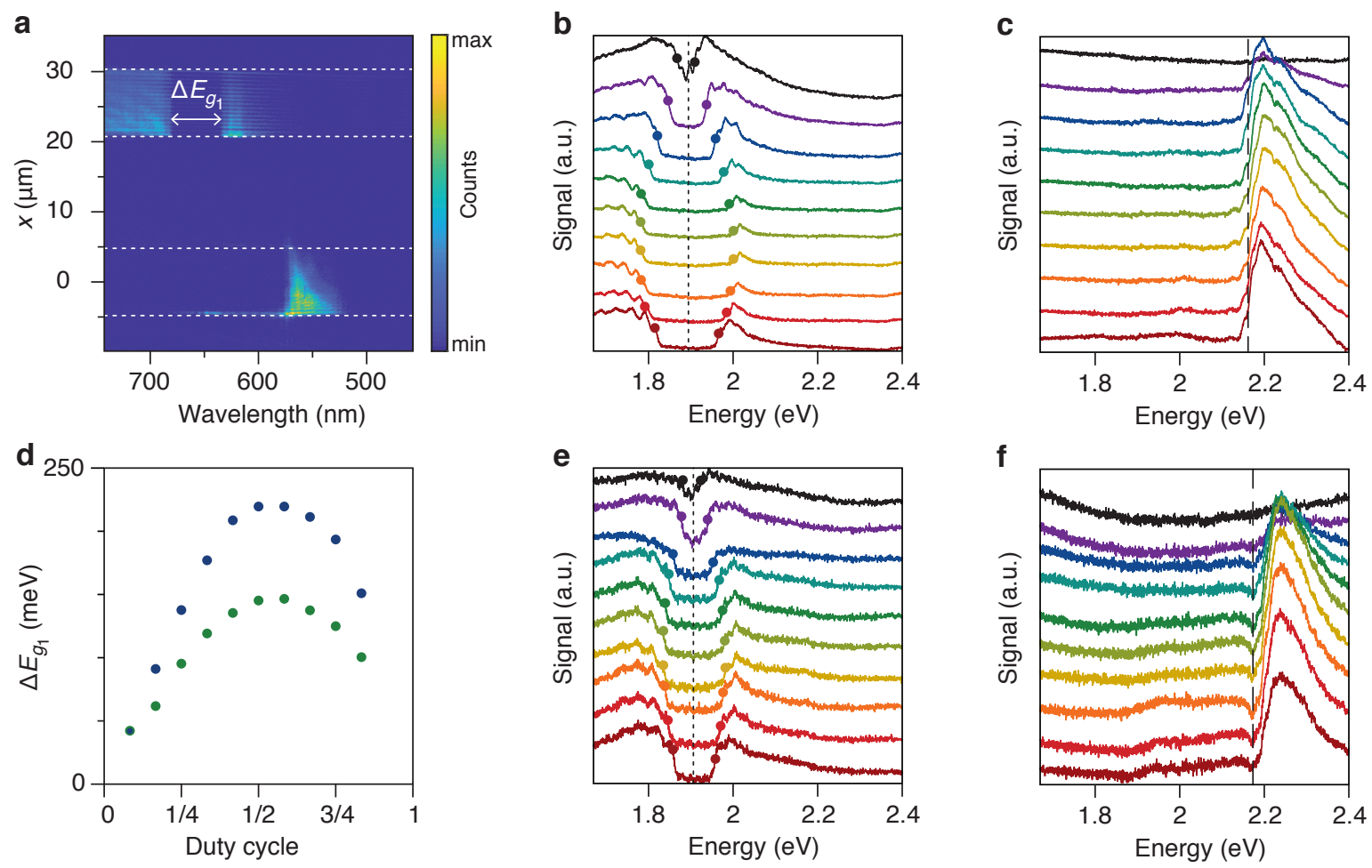

Figure S5. SPP-transmission measurements to probe the $g_{1}$ stop-gap width. (a) Example of a background-corrected position-resolved transmission spectrum for a feedback cavity with $h=19 \mathrm{~nm}$, $d=1 / 3$, and $p=280 \mathrm{~nm}$. SPPs were launched at an incoupling grating (at negative $x$; not shown), directed in the $+x$ direction through the feedback cavity (between -5 and $5 \mu \mathrm{m}$, indicated with dashed lines), and recollected at an outcoupling grating (between $\sim 20$ and $30 \mu \mathrm{m}$, indicated with dashed lines). The signal detected on the feedback cavity at wavelengths shorter than 580 nm stems from SPPs diffracted into the collection cone by $g_{1}$. (b) Spectra of signal transmitted through the feedback cavity and recollected from the outscattering grating and (c) spectra of the signal collected from the feedback cavity. The parameters of the feedback cavities were as in (a), but with duty cycles from 1/12 (top) to 10/12 (bottom) in steps of 1/12. The duty-cycle-dependent stop-gap width of the feedback cavities can be seen in (b) from the missing transmission signal around the center position of the $g_{1}$ stop gap (vertical dashed line, calculated with eq 2 of the main text). The vertical dashed line in (c) indicates the calculated onset of SPP-photon coupling by $g_{1}$ into the collection cone of the microscope objective under the assumption of an uncoupled dispersion. (d) Extracted $g_{1}$ stop-gap widths for feedback cavities with $h=19 \mathrm{~nm}$ (blue) and $h=13 \mathrm{~nm}$ (green). (e) and (f) as (b) and (c) but with $h=13 \mathrm{~nm}$. The optical setup is described in Section S3 above. 
a
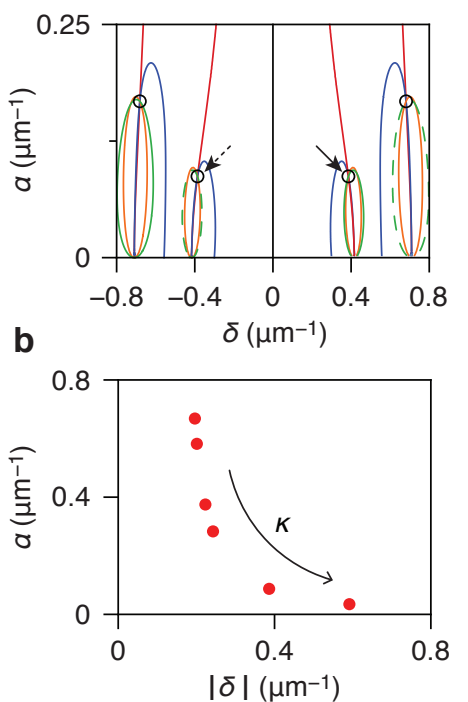

c

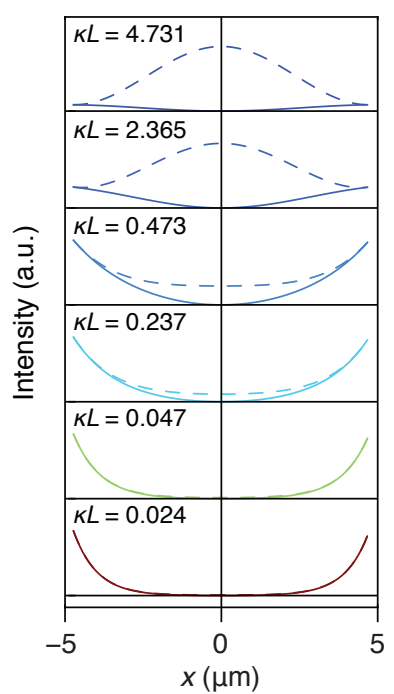

d

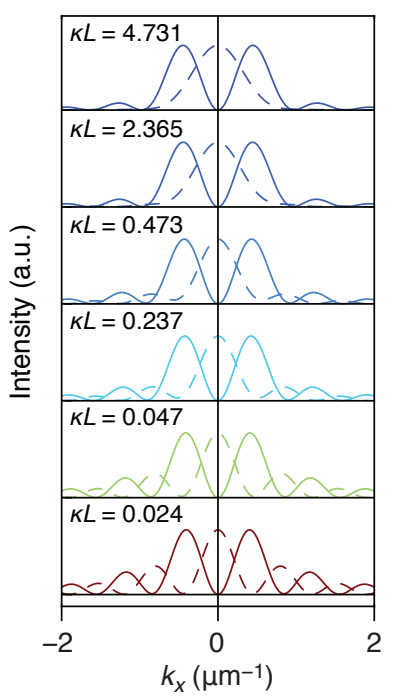

Figure S6. Modeling of the lasing-mode profiles in real- and $k$-space. (a) Solutions to eqs S8 (green solid line for the minus sign, green dashed line for the plus sign), S9 (red), S10 (blue), and S11 (orange) for the experimentally determined coupling strength of $\kappa=0.25 \mu \mathrm{m}^{-1}$ and a feedbackcavity length $L=9.46 \mu \mathrm{m}$. Self-consistent solutions can be found as the intersection points of the individual equations (black circles). The lowest-order lasing modes at both stop-gap edges (arrows) have a gain threshold of $\alpha=0.087 \mu \mathrm{m}^{-1}$ and detunings of $\delta= \pm 0.386 \mu \mathrm{m}^{-1}$. (b) Numerically found values for the gain threshold and detuning of the lowest-order lasing modes for coupling strengths $\kappa=\{0.0025,0.005,0.025,0.05,0.25,0.5\} \mu \mathrm{m}^{-1}$. The gain threshold decreases and the detuning increases with increasing coupling strength. (c) Real-space lasing-mode profiles as observed in the far field for the coupling strengths from (b) with increasing coupling strength from bottom to top. (d) Far-field lasing-mode profiles Fourier-transformed to $k$ space. Solid and dashed lines in (c) and (d) correspond to the solutions at the dark and bright stop-gap edge, respectively. For a fixed parameter set, the patterns at the two opposing stop-gap edges differ because of interference in the far field. 